\title{
A NOTE ON UNIQUENESS IN CLASSICAL ELASTODYNAMICS*
}

\author{
BY M. E. GURTIN AND ELI STERNBERG (Brown University)
}

Introduction. The conventional uniqueness theorems of classical elasticity theory, first established by Kirchhoff [1] ${ }^{1}$ for the equilibrium case and later extended to elastodynamics by Neumann [2], rest on the assumption that the elastic constants satisfy inequalities which are necessary and sufficient for the positive definiteness of the strainenergy density. The theorems referred to thus presuppose that

$$
\mu>0, \quad-1<\sigma<\frac{1}{2},
$$

if $\mu$ and $\sigma$ respectively denote the shear modulus and Poisson's ratio of the material.

Actual elastic materials obeying the linear isotropic stress-strain law conform to (1) and, in fact, fail to exhibit negative Poisson ratios. Nevertheless, the question as to whether conditions (1) are necessary for uniqueness, is of obvious theoretical interest. It is the purpose of the present note to show, with limitation to the first boundaryvalue problem of elastodynamics (surface displacements prescribed) and for bounded domains, that the inequalities (1) may be relaxed without loss of uniqueness. Specifically, we find that in this instance (1) may be replaced by the less stringent assumption

$$
\mu \geq 0, \quad-\infty<\sigma<\frac{1}{2}, \quad 1 \leq \sigma<\infty,
$$

or, equivalently, by

$$
c_{1}^{2}=\frac{\mu}{\rho} \geq 0, \quad c_{2}^{2}=\frac{2(1-\sigma) \mu}{(1-2 \sigma) \rho} \geq 0,
$$

in which $c_{1}$ and $c_{2}$ are the velocities of equivoluminal and irrotational waves in a medium of unlimited extent, whereas $\rho$ stands for the positive mass density. ${ }^{2}$ Accordingly, the generalized inequalities (2) admit a simple intrinsic interpretation: they require that the two basic wave-velocities of the elastic solid be real.

The foregoing extension of Neumann's theorem, which will be proved subsequently, is the dynamic counterpart of a known extension of the uniqueness theorem in elastostatics. The solution to the first boundary-value problem in the equilibrium theory is unique (under suitable regularity assumptions) if, instead of (1), one stipulates merely that

$$
\mu \neq 0, \quad-\infty<\sigma<\frac{1}{2}, \quad 1<\sigma<\infty .
$$

The relaxed inequalities (4) are evidently met provided the two wave velocities $c_{1}$ and $c_{2}$ are real and non-zero. For a bounded domain, this generalization of Kirchhoff's uniqueness theorem is implicit already in observations due to Kelvin [3]; it may also be inferred from available results appropriate to the Dirichlet problem for strongly

\footnotetext{
${ }^{*}$ Received December 15, 1960. The results communicated in this paper were obtained in the course of an investigation conducted under Contract Nonr-562(25) of Brown University with the Office of Naval Research in Washington, D. C.

${ }^{1}$ Numbers in brackets refer to the list of publications at the end of this paper.

${ }^{2}$ Actually, the inequalities (3) are sufficient for uniqueness in the present circumstances even if $\rho$ is negative.
} 
elliptic systems of partial differential equations, ${ }^{3}$ since (4) hold if and only if the displacement equations of equilibrium are of the strongly elliptic type. As shown by Duffin and Noll [6], the inequalities (4) assure the uniqueness of the solution to the first problem of elastostatics also for unbounded exterior domains, provided the displacements are required to vanish uniformly at infinity. Finally, it is clear from counter-examples due to Ericksen and Toupin [8] and Ericksen [9] that (4), in the equilibrium case, cannot be weakened further without loss of uniqueness.

A generalization of the uniqueness theorem in elastodynamics. The displacement equations of motion in the linear theory of homogeneous and isotropic elastic solids take the form

$$
\mu \nabla^{2} \mathbf{u}+\frac{\mu}{1-2 \sigma} \nabla \nabla \cdot \mathbf{u}+\mathbf{f}=\rho \frac{\partial^{2} \mathbf{u}}{\partial t^{2}},
$$

in which $\mathbf{u}(x, t)$ is the displacement vector ${ }^{5}$ and $\mathbf{f}(x, t)$ is the body-force intensity vector, the single argument $x$ representing the triplet of cartesian coordinates and $t$ denoting the time. Equation (5), which must hold throughout the region of space $D$ occupied by the medium, is subject to the initial conditions

$$
\mathbf{u}(x, 0)=\mathbf{u}^{0}(x),\left.\frac{\partial \mathbf{u}}{\partial t}\right|_{(x, 0)}=\mathbf{v}^{0}(x) \text { in } D,
$$

where $\mathbf{u}^{0}(x)$ and $\mathbf{v}^{0}(x)$ are the prescribed initial distributions of displacement and velocity. In the first problem of elastodynamics the accompanying boundary conditions are characterized by

$$
\mathbf{u}(x, t)=\mathbf{u}^{*}(x, t) \quad \text { on } B \quad(0<t<\infty),
$$

if $B$ is the boundary of $D$, while $\mathbf{u}^{*}(x, t)$ are the given surface displacements.

We now prove the following theorem. Let $D$ be a bounded regular region of space ${ }^{6}$ with the boundary $B$ and suppose that (3) hold. Then there exists at most one $\mathbf{u}(x, t)$, twice continuously differentiable for $\left(x_{1}, x_{2}, x_{3}\right)$ in $D+B(0 \leq t<\infty)$, which satisfies (5) in $D(0<t<\infty)$, and meets (6), (7).

In view of the linearity of (5), (6), (7) it is evidently sufficient to show that

$$
\mu \nabla^{2} \mathbf{u}+\frac{\mu}{1-2 \sigma} \nabla \nabla \cdot \mathbf{u}=\rho \frac{\partial^{2} \mathbf{u}}{\partial t^{2}} \text { in } D \quad(0<t<\infty),
$$

together with

$$
\begin{gathered}
\mathbf{u}(x, 0)=\left.\frac{\partial \mathbf{u}}{\partial t}\right|_{(x, 0)}=0 \text { in } D, \\
\mathbf{u}(x, t)=0 \text { on } B \quad(0<t<\infty),
\end{gathered}
$$

imply $\mathbf{u}(x, t)=0$ in $D(0<t<\infty)$.

Let $K(t)$ be the kinetic energy of the entire body, so that

$$
K(t)=\frac{\rho}{2} \int_{D}\left[\frac{\partial u}{\partial t}\right]^{2} d V
$$

${ }^{3}$ See Browder [4] and Morrey [5].

'See also [7].

${ }^{5}$ Letters in boldface designate vectors.

"The term "regular region of space" is used in the sense of Kellogg [10]. 
and define $G(t)$ through

$$
G(t)=\frac{\mu}{2} \int_{D}\left[\kappa(\nabla \cdot \mathfrak{u})^{2}+(\nabla \times \mathfrak{u})^{2}\right] d V, \quad \kappa=\frac{2(1-\sigma)}{1-2 \sigma} .
$$

From the divergence theorem, with the aid of (8), (11), (12), and elementary vectoranalytic identities, follows

$$
\mu \int_{B}\left[\kappa \frac{\partial \mathbf{u}}{\partial t} \nabla \cdot \mathbf{u}+\frac{\partial \mathbf{u}}{\partial t} \times(\nabla \times \mathbf{u})\right] \cdot \mathbf{n} d A=\frac{d K}{d t}+\frac{d G}{d t},
$$

where $\mathbf{n}$ is the outward unit normal of $B$.

According to (13) and (10),

$$
K(t)+G(t)=c \quad(0<t<\infty),
$$

in which $c$ is a constant. But by (11), (12), (9),

$$
K(0)=G(0)=0,
$$

whence, from (14) and continuity,

$$
K(t)+G(t)=0 \quad(0 \leq t<\infty) .
$$

Since $\kappa \geq 0$ by virtue of (3), both $K(t)$ and $G(t)$ are either non-negative or non-positive, so that (16) yields

$$
K(t)=0, \quad G(t)=0 \quad(0 \leq t<\infty) .
$$

On the other hand, the first of (17) in conjunction with (11) leads to

$$
\frac{\partial \mathbf{u}}{\partial t}=0 \quad \text { in } \quad D \quad(0 \leq t<\infty),
$$

from which, because of $(9), \mathfrak{u}(x, t)=\mathbf{u}(x, 0)=0$ in $D(0 \leq t<\infty)$. This completes the proof.

\section{References}

1. G. Kirchhoff, Ueber das Gleichgewicht und die Bewegung eines unendlich dünnen elastischen Stabes, J. für die reine und angewandte Mathematik 56, 285 (1859)

2. F. Neumann, Vorlesungen über die Theorie der Elasticität der festen Körper und des Lichtäthers, B. G. Teubner, Leipzig, 1885

3. W. Thomson, On the reflexion and refraction of light, Phil. Mag. 26, 414 (1888)

4. F. E. Browder, Strongly elliptic systems of differential equations, Ann. Math. Studies 33, 15 (1954)

5. C. B. Morrey, Jr., Second order elliptic systems of differential equations, Ann. Math. Studies 33, 101 (1954)

6. R. J. Duffin and W. Noll, On exterior boundary value problems in elasticity., Arch. Ratl. Mech. and Anal. 2, 2, 191 (1958)

7. M. E. Gurtin and E. Sternberg, On the first boundary value problem of linear elastostatics, Arch. Ratl. Mech. Anal. 6, 3, 177 (1960)

8. J. L. Ericksen and R. A. Toupin, Implications of Hadamard's condition for elastic stability with respect to uniqueness theorems, Can. J. Math. 8, 432 (1956)

9. J. L. Ericksen, On the Dirichlet problem for linear differential equations, Proc. Am. Math. Soc. 8, 3,521 (1957)

10. O. D. Kellogg, Foundations of potential theory, J. Springer, Berlin, 1929 